\title{
Simulation of giant tidal force of wormhole using curved optical spaces
}

\author{
R. Q. He, G. H. Liang, S. N. Zhu, and H. Liu ๑* \\ National Laboratory of Solid State Microstructures and School of Physics, Collaborative Innovation Center of Advanced Microstructures, \\ Nanjing University, Nanjing 210093 Jiangsu, China
}

(Received 16 August 2019; accepted 8 January 2020; published 2 March 2020)

\begin{abstract}
The tidal force of gravity is usually a weak force and is considered only in the movement of celestial bodies. However, in the presence of a very strong gravitational field, such as that of black holes and wormholes, tidal force also has an effect on light wave packets. In this work, we employ a curved optical waveguide to mimic the giant tidal force of wormholes on optical beams. We calculate the beam spreading caused by tidal force using general relativity. The theoretical calculations were in agreement with the experimental results. Our results show that the giant tidal force induces the divergence of optical deflection at the throat of a wormhole. This experimental system promises to be an effective methodology for the future studies mimicking gravitation.
\end{abstract}

DOI: 10.1103/PhysRevResearch.2.013237

\section{INTRODUCTION}

Although the detection of gravitational waves [1] and the imaging picture taken by the event horizon telescope [2] show remarkable achievement in observational astronomy, some of the unique properties of black holes are still very difficult to observe directly, such as Hawking radiation and its huge tidal force on light. The tidal force is a universal phenomenon that causes periodic fluctuations in the ocean's surface. It is caused by the gradient in the gravitational field, which is usually considered for movement of bodies with large size, such as celestial bodies. The effect of tidal force on light beams and wave packets is usually very weak and ignored since the gravitational field is almost homogenous in small scale. However, in the presence of a strong gravitational field, such as that of black holes and wormholes, the tidal force is very strong and even cannot be treated as a perturbation [3-5].

In order to investigate black holes experimentally, the idea of gravity analogy was proposed to stimulate them with precisely controlled table experiments under different laboratory conditions. Unruh was the first to propose the method of using acoustic waves in an accelerating fluid to mimic Hawking radiation near the event horizon [6]. Since then, the analogy of Hawking radiation has been realized in different experimental systems, such as superconducting circuits [7], Bose-Einstein condensates [8], superfluid ${ }^{3} \mathrm{He}$ A [9], Fermi degenerate flow [10], nonlinear optical fibers [11], and ions rotating on a ring [12]. Simultaneously, several other physicists also attempted to mimic wormholes in experimental systems such as acoustic wormholes [13], nanophotonic wormholes [14], plasmonic analog wormholes

\footnotetext{
*liuhui@nju.edu.cn

Published by the American Physical Society under the terms of the Creative Commons Attribution 4.0 International license. Further distribution of this work must maintain attribution to the author(s) and the published article's title, journal citation, and DOI.
}

[15], analog wormholes in graphene [16], and dc-SQUID (superconducting quantum interface device) arrays [17]. On the other hand, transformation optics based on metamaterials that manipulate permittivity and permeability profiles can be extensively investigated to design many artificial materials with novel optical applications [18-31]. By flexibly adjusting the electric and magnetic parameters, metamaterials can be used to simulate the metric of a gravitational field with very high precision [20]. With the help of transformation optics, black holes have been successfully mimicked in optical systems [32-35]. In addition, interesting phenomena such as wormholes [36], metric transitions [37,38], cosmological redshift [39], Einstein rings [40], Rindler space [41],uniform gravitational fields [42], and cosmic strings [43] were also observed. Recently, optical structures such as curved waveguides [44-47], nonlocal media [5,48], and optical lattices [49,50] have also been used to simulate these cosmic phenomena. One of the limitations of metamaterials that restricts us from exploring more complicated cases in gravitational fields is the demand for tremendous change in the refractive index, which requires a high level of material fabrication. To overcome this difficulty, physicists have turned to the use of two-dimensional (2D) embedded curved surfaces to mimic gravitational fields [44-47]. Such a method expands the experimental platform of analog gravity. By controlling the curvature of the surface, it is easier to realize analog space-time metrics that mimic extremely strong gravitational fields.

In this study, we will mimic giant tidal force of wormhole on optical beams using an embedded curved space. We have designed the sample based on the Morris-Thorne (MT) traversable wormhole metric. The sample was fabricated using a three-dimensional (3D) printing technique. Under different impact parameters, we measured the spreading of the light beam which is caused by tidal force. We also calculated the tidal force and the geodesic deviation based on general relativity. The theoretical results agree with the experimental results quite well. Our results show that the tidal force is very huge near the throat of the wormhole, which causes the beam to diverge completely at the throat. Such an effect destroys 

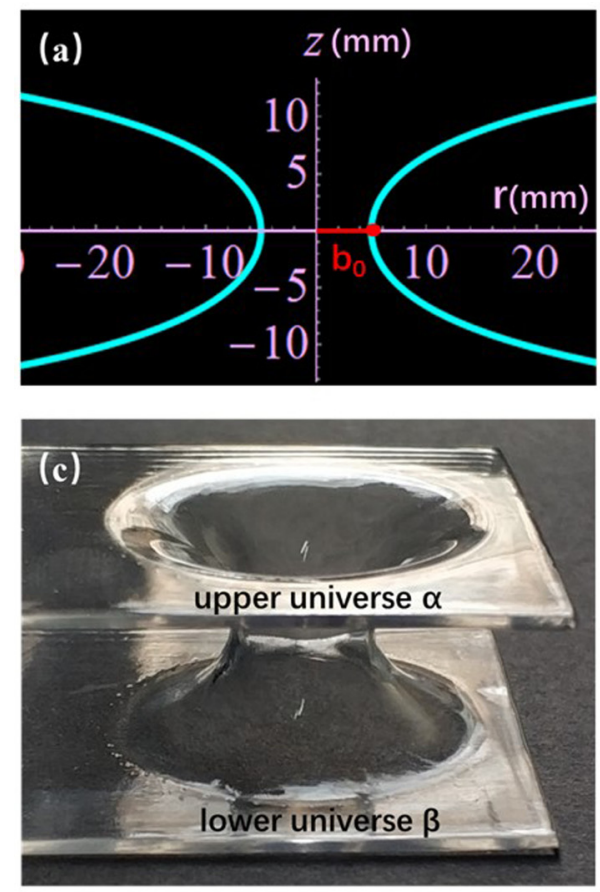
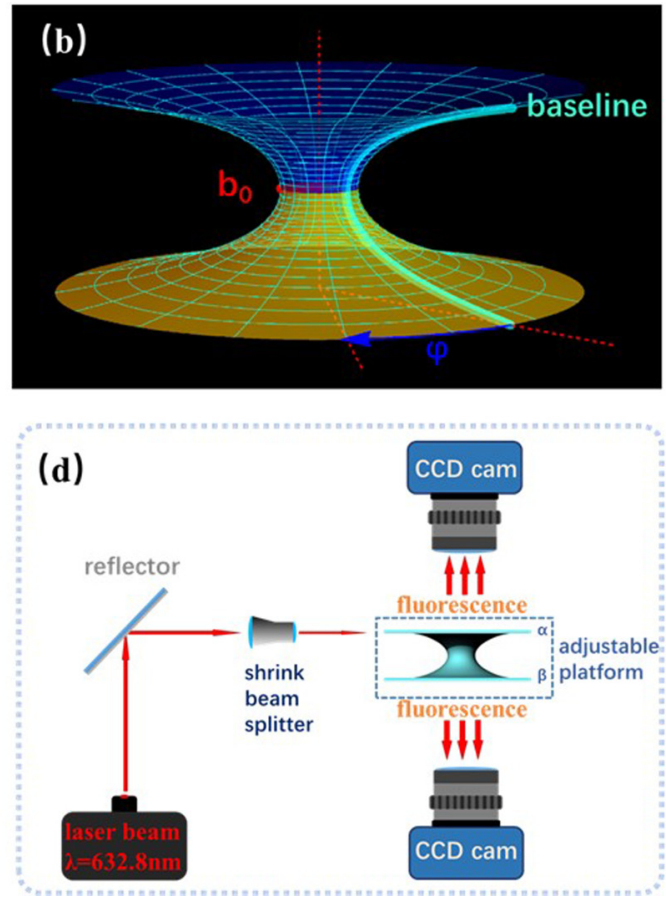

FIG. 1. (a) Rotation base line of the wormhole; (b) embedding diagram of the 2D wormhole; (c) image of the sample fabricated with 3D printing technique; and (d) optical experiment setup.

the propagation of the optical wave packet and also disrupts the optical information transfer.

\section{M-T METRIC EMBEDDED SPACE}

The MT metric was first proposed in 1988 to describe a traversable wormhole [51], which is given as a spherical coordinate system as follows:

$$
d s^{2}=-c^{2} d t^{2}+\frac{d r^{2}}{1-b_{0}^{2} / r^{2}}+r^{2}\left(d \theta^{2}+\sin ^{2} \theta d \varphi^{2}\right),
$$

where $c$ is the light speed and $b_{0}$ is the radius of the wormhole's throat. In a 2D static, the spherically symmetric solution of the project plane $(r, \varphi)$, the metric can be simplified to $d s^{2}=\frac{d r^{2}}{1-b_{0}{ }^{2} / r^{2}}+r^{2} d \varphi^{2}$. The intrinsic curvature of this $2 \mathrm{D}$ traversable wormhole is equivalent to a $2 \mathrm{D}$ embedding curved space in a 3D Euclidean space, with an extra dimension $d s^{2}=\left[1+\left(\frac{d z}{d r}\right)^{2}\right] d r^{2}+r^{2} d \varphi^{2}$. Here, the extra coordinate $Z$ is obtained from the MT metric as follows (see Appendix A for details):

$$
Z(r)= \pm b_{0} \ln \left(\frac{r}{b_{0}}+\sqrt{\left.\left(\frac{r}{b_{0}}\right)^{2}-1\right)} .\right.
$$

For $b_{0}=5 \mathrm{~mm}$, the function is given as a hyperbolic base line, as shown in Fig. 1(a). Due to the rotational symmetry of the MT wormhole metric, the embedded curved space can be obtained by rotating the base line around the $Z$ axis, shown as a hyperbolic surface in Fig. 1(b). In our experiment, a 3D printing technique is employed to fabricate a sample with uniform transparent resin with the thickness of $1 \mu \mathrm{m}$ based on the above design, as shown in Fig. 1(c). The transparent resin is Somos WC Ultra 10122, whose refractive index is 1.52 . The sample consists of the embedding hyperbolic curved space, as shown in Fig. 1(b). It is smoothly connected to two flat slabs, representing two flat spaces in the top plane $\alpha$ and the bottom plane $\beta$.

\section{GEODESIC TRAJECTORY CALCULATIONS AND EXPERIMENT RESULTS}

The Lagrangian for the gravitational field of a wormhole [52] can be expressed as $L=-c^{2} \dot{t}^{2}+\frac{r^{2}}{r^{2}-b_{0}^{2}} \dot{r}^{2}+r^{2} \dot{\varphi}^{2}$. Its geodesic line can be obtained as follows (see Appendix B for details):

$$
\left(\frac{d r}{d \varphi}\right)^{2}=\left(r^{2}-b_{0}^{2}\right)\left(\frac{r^{2}}{P^{2}}-1\right)
$$

where $P$ is the impact parameter, which is the perpendicular distance from the center of the wormhole to the incident light beam, and the solution to Eq. (3) is given. [See Appendix B for Fig. 6 and the solution to Eq. (3).]

Based on the impact parameter $P$, there can be three different situations. (1) When $P / b_{0}>1$, the calculated light rays do not pass through the wormhole. Three geodesic lines, 1, 2, and 3 , corresponding to $P / b_{0}=1.7,1.5$, and 1.3 , respectively, are shown in Fig. 2(a). In the figure, the black region is the wormhole and the blue background represents the outside space with a gravitational field. The light rays are represented by cyan lines with arrows marking the propagation directions. (2) When $P / b_{0}<1$, the calculated light rays pass through the wormhole. Three geodesic lines, 1, 2, and 3, corresponding to $P / b_{0}=0,0.3$, and 0.57 , respectively, are shown in Figs. 3(a) and 3(b). (3) When $P / b_{0}=1$, the calculated light rays approach the throat asymptotically and are finally trapped at the throat, which is shown as the yellow solid line 4 in Figs. 3(a) and 3(b). In all the three situations, the light rays 

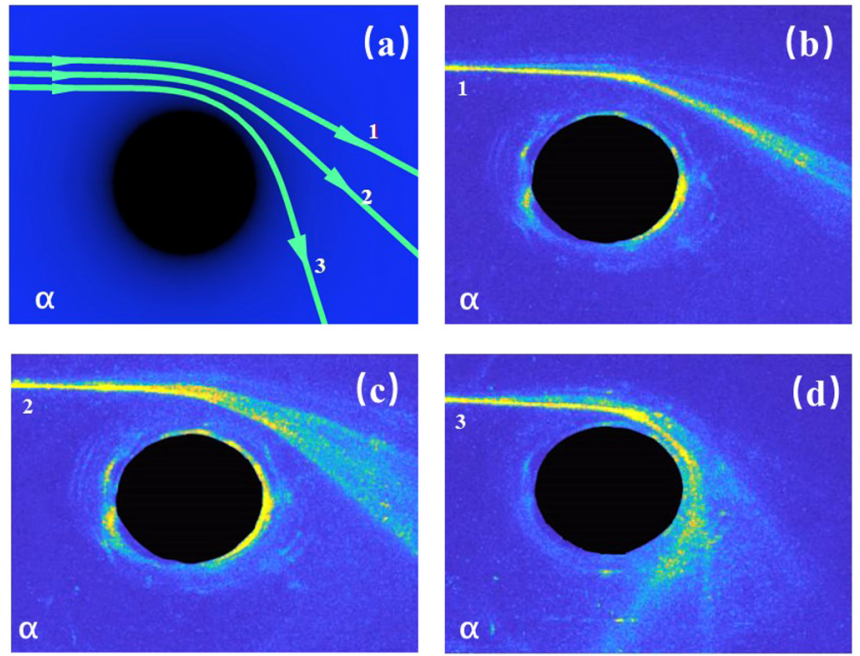

FIG. 2. (a) The calculated light geodesic lines with three impact parameters, $P / b_{0}=1.7,1.5$, and 1.3 ; experimental results for (b) $P / b_{0}=1.7$, (c) $P / b_{0}=1.5$, and (d) $P / b_{0}=1.3$.

are deflected by the gravitational field, and the deflection angle is defined as $\varphi$. The deflection angle $\varphi$ varies with the incident impact parameter $P$. Based on Eq. (3), we can calculate the dependence of $\varphi$ on $P$, which is given as a solid line in Fig. 4(a). The results show that for both $P / b_{0}>1$ and $P / b_{0}<1, \varphi$ increases as $P$ approaches $b_{0}$. At $P / b_{0}=1, \varphi$ diverges to infinity, which corresponds to the geodesic orbit at the throat of the wormhole, shown as yellow line 4 in Figs. 3(a) and 3(b).

The geodesic trajectory of a beam in the gravitational field of a wormhole can be simulated precisely in the experiment using the fabricated sample [Fig. 1(c)]. The experimental setup is shown in Fig. 1(d). In this experiment, we use a moving stage to hold the sample. A Gaussian beam is coupled to the $\alpha$ plane of the sample horizontally. Two cameras are used to take pictures of the optical beam inside the $\alpha$ plane (from above) and $\beta$ plane (from below). The moving stage can
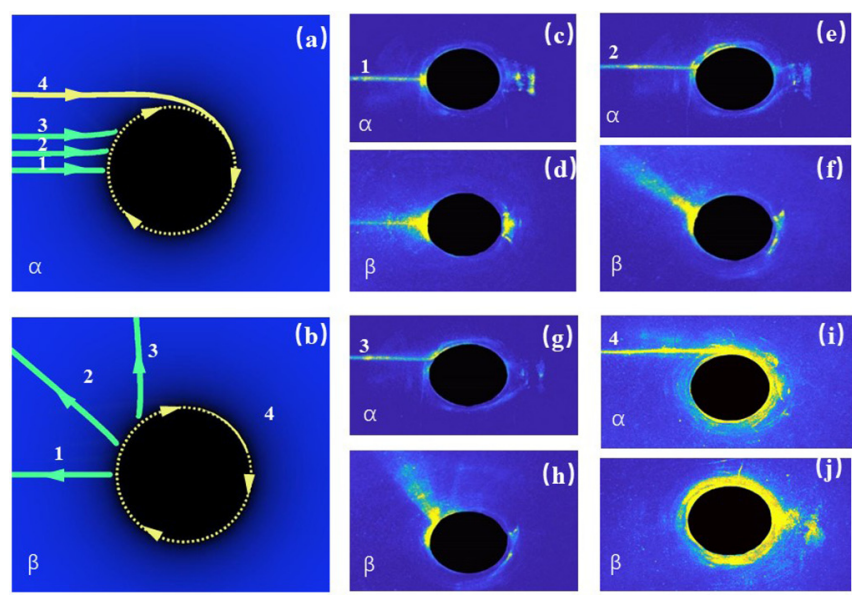

FIG. 3. The calculated light geodesic lines with four impact parameters $P / b_{0}=0,0.3,0.57$, and 1 in $\alpha$ plane (a) and $\beta$ plane (b); experimental results for $P / b_{0}=0$ (c), (d); $P / b_{0}=0.3$ (e), (f); $P / b_{0}=0.57(\mathrm{~g})$, (h); and $\mathrm{P} / b_{0}=1$ (i), (j).
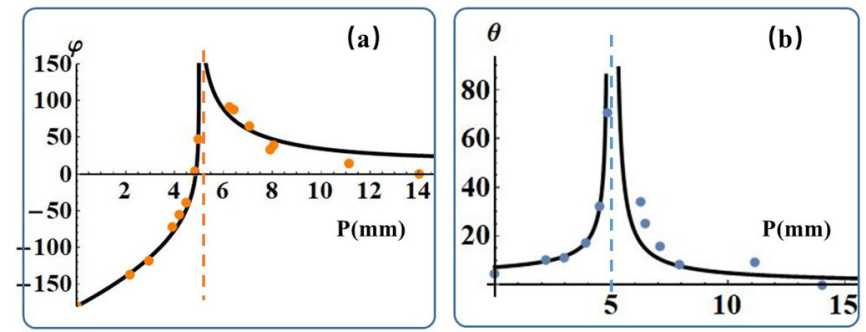

FIG. 4. (a) Deflection angle under different impact parameters: theoretical calculations (solid line) and optical measurements (origin dots). (b) Divergence angle under different impact parameters: theoretical calculations (solid line) and optical measurements (blue dots).

be controlled to change the incident location of beam, which enabled us to investigate the light trajectories under different impact parameters. The experimental process is provided in the attached movies. In order to compare the results of our experiment with those obtained theoretically, we chose $P / b_{0}=$ 0, 0.3, 0.57, 1, 1.3, 1.5, and 1.7, as shown in Figs. 2 and 3. Lines 1, 2, and 3 in Figs. 2(b)-2(d) correspond to the experimental results for $\frac{P}{b_{0}}=1.7,1.5$, and 1.3, respectively; the theoretical results are shown in Fig. 2(a). In good agreement with the calculations, all three rays are observed to not pass through the wormhole, and the middle trajectories of the beam are just bent with deflection angles $\varphi=27.97^{\circ}$, $44.57^{\circ}$, and $73.50^{\circ}$, respectively. In the process, the deflection angle $\varphi$ increases with the decrease in $P$. Lines 1,2 , and 3 in Figs. 3(c)-3(h) correspond to the experimental results for $\frac{P}{b_{0}}=0,0.3$, and 0.57 , respectively; the theoretical results are shown in Figs. 3(a) and 3(b). All three rays pass through the wormhole. They enter the wormhole through the $\alpha$ plane and exit through the $\beta$ plane. In the process, the rays are deflected, and the corresponding deflection angles of the middle trajectory obtained are $-180^{\circ},-139^{\circ}$, and $-93.55^{\circ}$. The experimental results agree with the theoretical calculations shown in Figs. 3(a) and 3(b). The experimental result corresponding to the critical case $P / b_{0}=1$ is shown in Figs. 3(i) and 3(j). The rays are trapped within the stable orbit at the throat of the wormhole, which is also in agreement with the theoretical line 4. In Figs. 2 and 3, we give only some special cases of light rays. However, experimentally, we have done measurements of many different impact parameters. These results are shown as orange dots in Fig. 4(a). Except for the deviation at large impact parameters caused by fabrication error of the throat of the wormhole, the measured dots agree with the theoretical solid line quite well.

\section{TIDAL FORCE}

In the above experiment, the beam is approximately regarded as a bunch of ideal trajectory lines with no wave properties. In some situations, such an approximation is not good enough. For example, the light beam cannot be simply regarded as ideal geodesic trajectories but as wave packets in space-time instead. As we know, any object with geometry size, when placed in an inhomogeneous gravitational field, would be subject to tidal force by gravity, and optical wave 

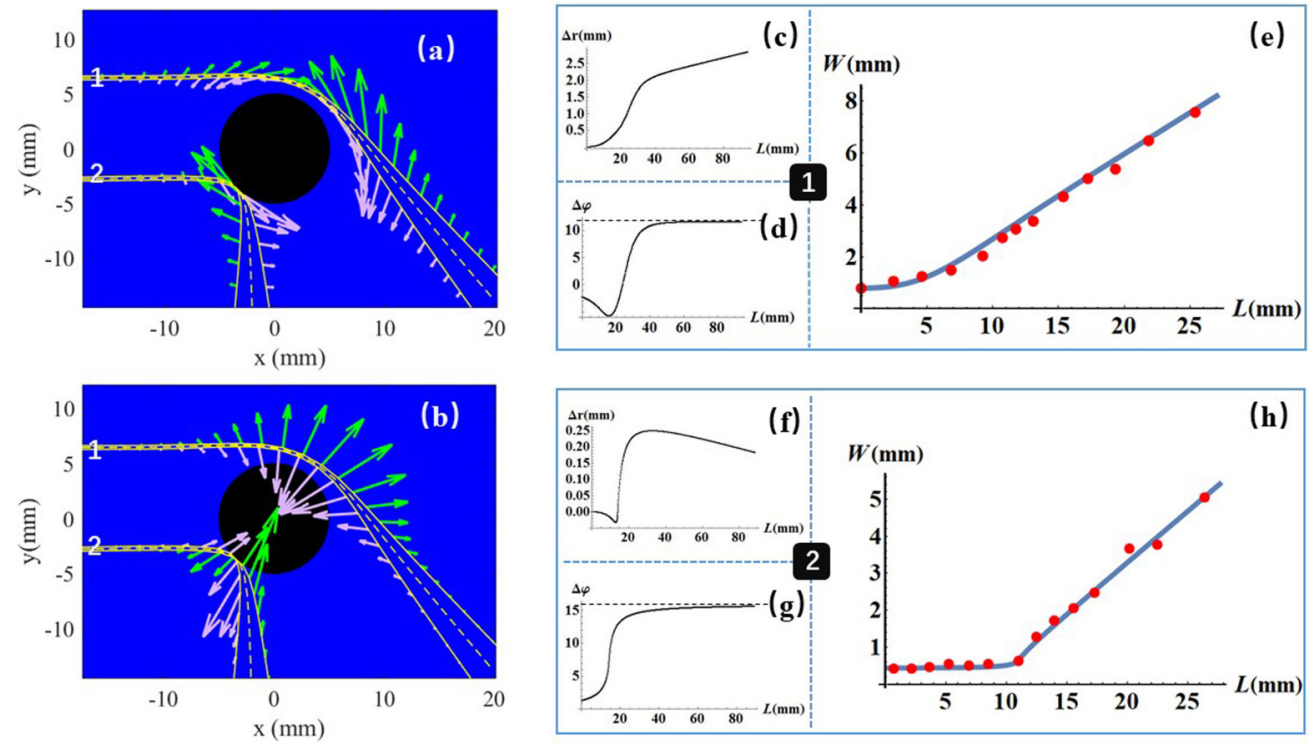

FIG. 5. The change of acceleration (a) $a_{r}$ and (b) $a_{\varphi}$ along two geodesic lines 1 and 2; the change of geodesic deviation (c) $\Delta r$, (d) $\Delta \varphi$, and (e) beam width along two geodesic lines 1; the change of geodesic deviation (f) $\Delta r,(\mathrm{~g}) \Delta \varphi$, and (h) beam width along two geodesic lines 2 .

packets are no exception. One of the research studies used the photothermal effect to mimic the weak tidal force on optical packets. The results show that the tidal force induces a redshift of the optical packets [5]. The tidal effect is very weak and does not produce a remarkable change on the light beam. As is known, the greater the gradient of the gravitational field, the stronger the tidal force that will be exerted. When the tidal force is strong enough, the object will be torn to pieces. Usually, the tidal forces of celestial bodies on wave packets are very small and can be ignored [just like the behavior of the beam far away from the throat in Fig. 2(b)]. However, in the case of the strong gravitational field of wormholes, since the gradient is very large, its tidal force on wave packets will be very high [see Fig. 2(d) and Figs. 3(e)-3(h)]. Such a giant tidal force on wave packets is hard to observe on Earth. Besides, at present, it is impossible for anybody go to a wormhole to observe this giant tidal force. However, the optical wormhole setup in this work can be used to stimulate such giant tidal forces on optical wave packets inside wormholes.

A careful observation of the images shown in Figs. 2(b)2(d) and 3(c)-3(j) reveals that when the optical beams pass through the gravitational field of the wormhole, an obvious beam spreading occurs. It is worth stressing that the beam is not uniformly spreading but torn into bunches with a varying distribution of intensity because of the effective gravitational field, which means the Gaussian beam cannot maintain its shape but changes into new forms in the evolution process around the wormhole. Although an optical beam will spread even in free space without gravity, it is a very slow process. The beam spreading in our experiment is much faster than that in free space because of the gravitational field gradient. Besides, when the beam is closer to the wormhole, the beam spreading will be higher and shows a different intensity distribution. Such effects can be carefully analyzed based on our theoretical model. To investigate the spreading of the optical beam, we define the width of the beam with its two edges as shown in Fig. 7 in Appendix $C$ (see Appendix C for Fig. 7). The impact parameter of the beam center is $P$, and the two edges enter the gravitational field with different incident impact parameters, $P_{1}$ and $P_{2}$. The difference between the impact parameters, $\Delta P=P_{1}-P_{2}$, is defined as the width of the beam. The two edges propagate along two different geodesic trajectories. Their deflection angles, $\varphi_{1}$ and $\varphi_{2}$, can be calculated using Eq. (3). We obtain the divergence angle $\theta=\varphi_{1}-\varphi_{2}$ of the optical beam after it leaves the wormhole. For instance, we choose the beam width $\Delta p=0.4 \mathrm{~mm}$ and calculate the divergence angle under different impact parameters, which is shown as a solid line in Fig. 4(b). The result shows that when $P$ is much larger or smaller than $b_{0}, \theta$ is small and the beam broadening is also small. When $P$ approaches $b_{0}, \theta$ is increases very quickly and the beam will diverge at $P=b_{0}$. The divergence angle of the beam can be obtained from the experimental results shown in Figs. 2 and 3. These results are provided as blue dots in Fig. 4(b), which agrees well with the theoretically calculated solid line.

To approximately describe this effect, we give the theoretical geodesic deviation in comparison with the experiment results. (Note that in this article we apply this theory only as an approximation, while the accurate intensity distribution might be obtained by a great deal of calculation.) According to Einstein's theory of general relativity, the tidal force of an inhomogeneous gravitational field can be described using the acceleration $\left(a_{r}, a_{\varphi}\right)$ obtained from geodesic deviation $(\Delta r, \Delta \varphi)$ (see Appendix D for details):

$$
\begin{aligned}
& a_{r}=-\frac{b_{0}^{2}}{r^{2}}\left[\Delta \varphi \frac{d r}{d \lambda} \frac{d \varphi}{d \lambda}-\left(\frac{d \varphi}{d \lambda}\right)^{2} \Delta r\right], \\
& a_{\varphi}=-\frac{b_{0}^{2}}{b_{0}^{2} r^{2}-r^{4}}\left[\Delta r \frac{d r}{d \lambda} \frac{d \varphi}{d \lambda}-\Delta \varphi\left(\frac{d r}{d \lambda}\right)^{2}\right] .
\end{aligned}
$$


From Eq. (4), we can calculate the change in acceleration $a_{\varphi}$ and $a_{r}$ along geodesic lines 1 and 2, which are shown as arrows in Figs. 5(a) and 5(b). Here, the magnitude of acceleration is represented by the length of the arrows. In both cases, the tidal force increases when the beam approaches the wormhole and decreases when it leaves the wormhole. The tidal force is very high near the wormhole, which causes an abrupt broadening of the beam, as shown in Figs. 5(e) and 5(h). A comparison between the theoretical and experimental results shows that the tidal force is the reason for the divergence of the optical beam. The divergence of the beam observed in our experiment can be seen as a good simulation of the giant tidal force on optical wave packets by wormholes.

\section{CONCLUSION AND OUTLOOK}

As we know, in free space, optical information is transferred through wave packets. The spreading of wave packets will influence the information transfer process. Ideally, we hope that the optical wave packet does not spread and that it can support efficient information transfer. Usually, in the presence of a gravitational field, the tidal force is very small and does not change the wave packet. The spreading of wave packets is mainly caused by the diffraction of beams and has nothing to do with gravity. However, our study shows that, in the presence of a very high gravitational field of the wormhole, the tidal effect cannot be ignored and the huge tidal force will cause the spreading of the wave packets or even destroy the wave packet very quickly. This disrupts the optical information transfer when wave packets pass through the gravitational field of wormholes.

Our study shows that tidal force cannot be ignored anymore when we investigate the information technique with black holes or wormholes in the future. In summary, we employ a 2D curved space to simulate the giant tidal force of wormholes on optical wave packets. Our theoretical and experimental results show that the tidal force will cause the spreading and distortion of wave packets. Although traversable wormholes are often imaged to work as a space-time tunnel for interstellar travel in the future, our study shows that a huge tidal force will have a destructive effect. The giant tidal force not only tears the matter which passes through a wormhole but also disrupts the optical information transfer. Therefore, tidal force is an important issue which cannot be ignored in the relativistic information technique. Our study provides a good experimental platform to simulate the tidal force in the presence of a strong gravitational field.

\section{ACKNOWLEDGMENTS}

This work was financially supported by the National Natural Science Foundation of China (Grants No. 11690033, No. 61425018, No. 11621091, and No. 11704181), National Key R\&D Program of China (Grant No. 2017YFA0303702), and National Key Research and Development Program of China (Grant No. 2017YFA0205700).

\section{APPENDIX A: MORRIS-THORNE METRIC AND EMBEDDING DIAGRAM}

Since the introduction of the Einstein-Rosen bridge, several types of wormholes have been suggested. One of the simplest metrics of a wormhole, proposed by Morris and Thorne, is given as

$$
d s^{2}=-c^{2} d t^{2}+\frac{d r^{2}}{1-b_{0}^{2} / r^{2}}+r^{2}\left(d \theta^{2}+\sin ^{2} \theta d \varphi^{2}\right),
$$

where $t$ is the global time, $(r, \theta, \varphi)$ are coordinates in the spherical system, $b_{0}$ is the throat radius, and $c$ is the speed of light. Because of the spherical symmetry of the static metric, we can only consider a two-dimensional (2D) hypersurface with $t=$ constant and $\theta=\pi / 2$, in order to manifest the property of a wormhole. The metric can then be simplified as

$$
d s^{2}=\frac{d r^{2}}{1-b_{0}^{2} / r^{2}}+r^{2} d \varphi^{2} .
$$

This metric shows an apparent axial symmetry which enables us to construct a rotational surface as a $2 \mathrm{D}$ embedding diagram in a $3 \mathrm{D}$ Euclidean space with an extra coordinate $z$. The rotational surface equation can be described by cylindrical coordinates as

$$
d s^{2}=d r^{2}+d z^{2}+r^{2} d \phi^{2} .
$$

Considering $z$ as a function of $r$, the equation may be rewritten as

$$
d s^{2}=\left[1+\left(\frac{d z}{d r}\right)^{2}\right] d r^{2}+r^{2} d \varphi^{2} .
$$

Combing Eqs. (2) and (4), we can obtain $z(r)$ as follows:

$$
z(r)= \pm b_{0} \ln \left[\frac{r}{b_{0}}+\sqrt{\left(\frac{r}{b_{0}}\right)^{2}-1} .\right.
$$

Now we are able to plot the embedding diagram of the MT wormhole, which is formed by rotating the base line $z(r)$.

\section{APPENDIX B: GEODESIC LINE AND THE DEFLECTION ANGLE}

The Lagrangian is given as

$$
L=g_{\mu \nu} \dot{x}^{\mu} \dot{y}^{\nu}, \quad \dot{x}^{\mu}=\frac{d x^{\mu}}{d \lambda},
$$

where $\lambda$ is an affine parameter and $x^{\mu}=(t, r, \theta, \varphi)$. We then get the Euler-Lagrangian equation:

$$
\frac{d}{d \lambda} \frac{\partial L}{\partial \dot{x}^{\mu}}-\frac{\partial L}{\partial x^{\mu}}=0 .
$$

For a wormhole metric in Eq. (A2), the Lagrangian is

$$
L=-c^{2} \dot{t}^{2}+\frac{r^{2}}{r^{2}-b_{0}^{2}} \dot{r}^{2}+r^{2} \dot{\varphi}^{2} .
$$

From the Euler-Lagrangian equation or the geodesic equation, we obtain two constants of motion from the conservation of energy and angular momentum, namely,

$$
c^{2} \dot{t}=k, \quad r^{2} \dot{\varphi}=h .
$$

Substituting the two constants in Eqs. (B2) and (B3), we can easily obtain the null geodesic equation as below:

$$
\dot{r}^{2}=\left(1-\frac{b_{0}^{2}}{r^{2}}\right)\left(\frac{k^{2}}{c^{2}}-\frac{h^{2}}{r^{2}}\right) \text {. }
$$




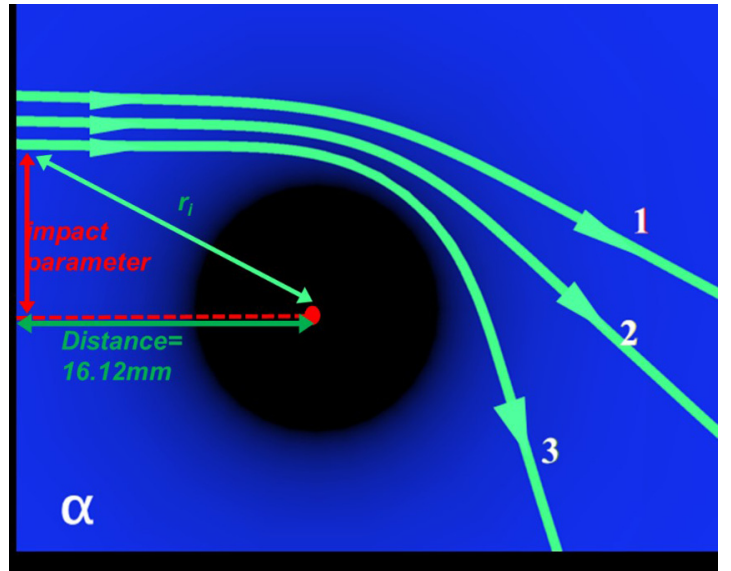

FIG. 6. Illustration of geodesic lines, impact parameter, and initial distance.

In order to obtain a solution function $r(\varphi)$, Eq. (B5) may be rewritten as

$$
\begin{aligned}
\left(\frac{d r}{d \varphi}\right)^{2} & =\left(r^{2}-b_{0}^{2}\right)\left(\frac{k^{2} r^{2}}{h^{2} c^{2}}-1\right) \\
& =\left(r^{2}-b_{0}^{2}\right)\left(\frac{c^{2}}{r^{2}\left(\frac{d \varphi}{d t}\right)^{2}}-1\right),
\end{aligned}
$$

where $\frac{k}{h}=\frac{c^{2}}{r^{2}\left(\frac{d \varphi}{d t}\right)}$. We define the impact parameter $P$, which is the perpendicular distance from the center of the wormhole to the incident light beam, as shown in Fig. 6. If the impact parameter $P$ is defined at a location very far from the wormhole, we will have $r\left(\frac{d \varphi}{d t}\right)=c \frac{P}{r}$; we then obtain

$$
\left(\frac{d r}{d \varphi}\right)^{2}=\left(r^{2}-b_{0}^{2}\right)\left(\frac{r^{2}}{P^{2}}-1\right) .
$$

Considering different impact parameters in Eq. (B7), we have three different situations below:

Case 1 . If $P / b_{0}>1$. In this case, the geodesic line will not pass through the wormhole; it is just deflected by the wormhole. In this case, we have the solutions of Eq. (B7),

$$
\varphi_{1}= \pm\left[F(r, P)-F\left(r_{i}, P\right)\right],
$$

where the function $F$ is defined as $F(r, P)=$ $\int_{0}^{P / r} \frac{d x}{\sqrt{\left(1-x^{2}\right)\left[1-\left(b_{0} / P\right)^{2} x^{2}\right]}}$. In Fig. 6, the impact parameter is defined at the incident location, $D=16.12 \mathrm{~mm}$. The distance of the incident location is $r_{i}=\sqrt{P^{2}+D^{2}}$. In this case, we can calculate the deflection angle of the geodesic line using $r \rightarrow \infty$ as

$$
\varphi_{1}^{\infty}=2 K(P)-F\left(r_{i}, P\right),
$$

where $K(P)$ is defined as $K(P)=\int_{0}^{1} \frac{d x}{\sqrt{\left(1-x^{2}\right)\left[1-\left(b_{0} / P\right)^{2} x^{2}\right]}}$.
Case 2. If $P / b_{0}<1$, the geodesic line will pass through the wormhole from $\alpha$ universe to $\beta$ universe. In this case,

$$
\varphi_{2}= \pm\left[G(r, P)-G\left(r_{i}, P\right)\right],
$$

where $G(r, P)$ is defined as $G(r, P)=$ $\int_{0}^{b_{0} / r} \frac{d x}{\sqrt{\left(1-x^{2}\right)\left[1-\left(P / b_{0}\right)^{2} x^{2}\right]}}$. In this case, we calculate the deflection angle of the geodesic line using $r \rightarrow \infty$ as

$$
\varphi_{2}{ }^{\infty}=2 H(P)-\frac{P}{b_{0}} G\left(r_{i}, P\right),
$$

where $H(P)$ is defined as $H(P)=\int_{0}^{1} \frac{d x}{\sqrt{\left(1-x^{2}\right)\left[1-\left(P / b_{0}\right)^{2} x^{2}\right]}}$.

Case 3. If $P / b_{0}=1$, the solution $\varphi$ of Eq. (12) diverges. The geodesic line keeps circling around the wormhole, and the beam is trapped inside a stable orbit. Using Eqs. (B8) and (B10), the geodesic lines corresponding to $P / b_{0}=0$, $0.3,0.57,1.0,1.3,1.5$, and 1.7 are calculated as shown in Figs. 2(a), 3(a), and 3(b). Using Eqs. (B9) and (B11), the deflection angles corresponding to $P / b_{0}=0,0.3,0.57,1.3$, 1.5, and 1.7 are calculated and shown in Fig. 4(a).

\section{APPENDIX C: DIVERGENCE ANGLE OF THE OPTICAL BEAM}

Experimentally, we notice that the deflection angle of the light beam changes with the impact parameter $P$. Accordingly, the divergence angle of the light beam can be calculated as $\theta(P)=\varphi^{\infty}(y+\Delta P)-\varphi^{\infty}(P)=\Delta P \cdot d \varphi^{\infty} / d P$. Note that we assume that the source of the light beam is far away from the throat; thus, approximately we have $r_{i} \gg b_{0}$. With this approximation, when the light beam does not pass through the wormhole, we have $\frac{d \varphi_{1}^{\infty}}{d P}=\frac{1}{P-b_{0}} E(P)-\frac{1}{P} H(P)$, where $E(P)=\int_{0}^{1} \frac{\sqrt{\left[1-\left(b_{0} / P\right)^{2} x^{2}\right]}}{\sqrt{\left(1-x^{2}\right)}} d x$. We then obtain the divergence angle as below:

$$
\theta=\frac{\Delta P}{P-b_{0}} E(P)-\frac{\Delta P}{P} H(P) .
$$

When the light beam passes through the wormhole, we have $\frac{d \varphi_{2}^{\infty}}{d P}=\frac{1}{b_{0}-P} I(P)+\frac{1}{b_{0}} H(P)$, where $I(P)$ is expressed as $I(P)=\int_{0}^{1} \frac{\sqrt{\left[1-\left(P / b_{0}\right)^{2} x^{2}\right]}}{\sqrt{\left(1-x^{2}\right)}} d x$. We then can obtain the divergence angle as below:

$$
\theta=\Delta p \frac{d \varphi_{2}^{\infty}}{d P}=\frac{\Delta p}{b_{0}-P} I(P)+\frac{\Delta p}{b_{0}} H(P) .
$$

The divergence angles are calculated based on Eqs. (B2) and (B3), and compared with the experimental values shown in Fig. 4(b).

\section{APPENDIX D: TIDAL FORCE}

For vector $A^{\mu}$, the second derivation along a geodesic line is is

$$
\begin{aligned}
\frac{D^{2} A^{\mu}}{D \lambda^{2}} & =\frac{D}{D \lambda}\left(\frac{D A^{\mu}}{D \lambda}\right)=\frac{d}{d \lambda}\left(\frac{D A^{\mu}}{D \lambda}\right)+\Gamma^{\mu}{ }_{\alpha \beta} \frac{D A^{\alpha}}{D \lambda} \frac{d x^{\beta}}{d \lambda}=\frac{d}{d \lambda}\left(\frac{d A^{\mu}}{d \lambda}+\Gamma^{\mu}{ }_{\alpha \beta} A^{\alpha} \frac{d x^{\beta}}{d \lambda}\right)+\Gamma^{\mu}{ }_{\alpha \beta}\left(\frac{d A^{\alpha}}{d \lambda}+\Gamma^{\alpha}{ }_{\kappa \lambda} A^{\kappa} \frac{d x^{\lambda}}{d \lambda}\right) \frac{d x^{\beta}}{d \lambda} \\
& =\frac{d^{2} A^{\mu}}{d \lambda^{2}}+\Gamma^{\mu}{ }_{\alpha \beta, \nu} \frac{d x^{\mu}}{d \lambda} A^{\alpha} \frac{d x^{\beta}}{d \lambda}+2 \Gamma^{\mu}{ }_{\alpha \beta} \frac{d A^{\mu}}{d \lambda} \frac{d x^{\beta}}{d \lambda}-\Gamma^{\mu}{ }_{\alpha \beta} A^{\alpha} \Gamma^{\beta}{ }_{\kappa \lambda} A^{\kappa} \frac{d x^{\kappa}}{d \lambda} \frac{d x^{\lambda}}{d \lambda}+\Gamma^{\mu}{ }_{\alpha \beta} \Gamma^{\alpha}{ }_{\kappa \lambda} A^{\kappa} \frac{d x^{\lambda}}{d \lambda} \frac{d x^{\beta}}{d \lambda}
\end{aligned}
$$


Assuming that the position vector of two beams is expressed as $x^{\mu}(\tau)$ and $x^{\mu}(\tau)+s^{\mu}(\tau)$, we write out their geodesic line equations as below:

$$
\begin{aligned}
& \frac{d^{2} x^{\mu}}{d \tau^{2}}+\Gamma_{\alpha \beta}^{\mu}(x) \frac{d x^{\alpha}}{d \tau} \frac{d x^{\beta}}{d \tau}=0, \\
& \frac{d^{2}\left(x^{\mu}+s^{\mu}\right)}{d \tau^{2}}+\Gamma_{\alpha \beta}^{\mu}(x+s)\left(\frac{d x^{\alpha}}{d \tau}+\frac{d s^{\alpha}}{d \tau}\right)\left(\frac{d x^{\beta}}{d \tau}+\frac{d s^{\beta}}{d \tau}\right)=0 .
\end{aligned}
$$

If the two beams are initially closed enough, $s^{\mu}$ and $d s^{\mu} / d \lambda$ are infinitesimally small, approximately leading to $\Gamma_{\alpha \beta}^{\mu}(x+s) \cong \Gamma_{\alpha \beta}^{\mu}(x)+\Gamma_{\alpha \beta, \sigma}^{\mu} s^{\sigma}$. From Eq. (D2), we obtain

$$
\frac{d^{2} s^{\mu}}{d \tau^{2}}=-\Gamma_{\alpha \beta, \sigma}^{\mu} s^{\sigma} \frac{d x^{\alpha}}{d \tau} \frac{d x^{\beta}}{d \tau}-2 \Gamma^{\mu}{ }_{\alpha \beta}(x+s) \frac{d s^{\alpha}}{d \tau} \frac{d x^{\beta}}{d \tau} .
$$

Treating $s^{\mu}$ as $A^{\mu}$ and substituting Eqs. (D3) in (D1), we can get the second derivative of $s^{\mu}$ along the geodesic line,

$$
\frac{D^{2} s^{\mu}}{D \lambda^{2}}=R_{\alpha \beta \sigma}^{\mu} \frac{d x^{\alpha}}{d \lambda} \frac{d x^{\beta}}{d \lambda} s^{\sigma},
$$

where $R_{\alpha \beta \sigma}^{\mu}$ is the Riemann tensor, which can be expressed as

$$
\begin{aligned}
& R_{r \varphi r}^{\varphi}=\frac{b_{0}^{2}}{b_{0}^{2} r^{2}-r^{4}}, \quad R_{\mathrm{rr} \varphi}^{\varphi}=-\frac{b_{0}^{2}}{b_{0}^{2} r^{2}-r^{4}}, \\
& R_{\varphi r \varphi}^{\varphi}=-\frac{b_{0}^{2}}{r^{2}}, \quad R_{\varphi \varphi r}^{r}=\frac{b_{0}^{2}}{r^{2}} .
\end{aligned}
$$

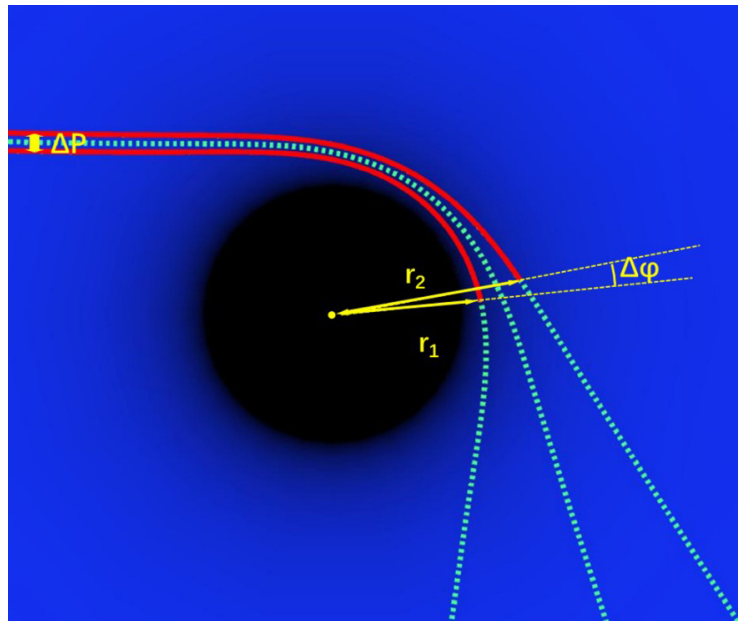

FIG. 7. Illustration of spreading of the optical beam.

Accordingly, for the metric of the MT wormhole, the relative accelerations are calculated as

$$
\begin{gathered}
a^{r}=-\frac{b_{0}^{2}}{r^{2}}\left[\Delta \varphi \frac{d r}{d \lambda} \frac{d \varphi}{d \lambda}-\left(\frac{d \varphi}{d \lambda}\right)^{2} \Delta r\right], \\
a^{\varphi}=-\frac{b_{0}^{2}}{b_{0}^{2} r^{2}-r^{4}}\left[\Delta r \frac{d r}{d \lambda} \frac{d \varphi}{d \lambda}-\Delta \phi\left(\frac{d r}{d \lambda}\right)^{2}\right],
\end{gathered}
$$

where $\Delta r=r_{1}(\lambda)-r_{2}(\lambda)$ and $\Delta \varphi=\varphi_{1}(\lambda)-\varphi_{2}(\lambda)$, with subscripts 1 and 2 denoting two closed geodesics. The illustration of the polar coordinate is shown in Fig. 7. From the geodesic equation in the polar coordinate system, we calculate the change $(\Delta r, \Delta \varphi)$ along the geodesic line, as shown in Figs. 5(c), 5(d), 5(f), and 5(g). We then calculate the change in the tidal acceleration $\left(a^{r}, a^{\varphi}\right)$ along the geodesic lines, as shown in Figs. 5(a) and 5(b).
[1] B. P. Abbott, R. Abbott, T. D. Abbott, M. R. Abernathy, F. Acernese, K. Ackley, C. Adams, T. Adams, P. Addesso, R. $\mathrm{X}$. Adhikari et al. (LIGO Scientific Collaboration and Virgo Collaboration), Phys. Rev. Lett. 116, 061102 (2016).

[2] K. Akiyama, A. Alberdi, W. Alef, K. Asada, R. Azulay, A.-K. Baczko, D. Ball, M. Baloković, J. Barrett, D. Bintley et al. (The Event Horizon Telescope Collaboration), Astrophys. J. Lett. 875, L4 (2019).

[3] B. Mashhoon and D. S. Theiss, Phys. Rev. Lett. 49, 1542 (1982).

[4] C. Chicone and B. Mashhoon, Classical Quant. Grav. 23, 4021 (2006).

[5] R. Bekenstein, R. Schley, M. Mutzafi, C. Rotschild, and M. Segev, Nat. Phys. 11, 872 (2015).

[6] W. G. Unruh, Phys. Rev. Lett. 46, 1351 (1981).

[7] P. D. Nation, M. P. Blencowe, A. J. Rimberg, and E. Buks, Phys. Rev. Lett. 103, 087004 (2009).

[8] L. J. Garay, J. R. Anglin, J. I. Cirac, and P. Zoller, Phys. Rev. Lett. 85, 4643 (2000)

[9] T. A. Jacobson and G. E. Volovik, Phys. Rev. D 58, 064021 (1998).
[10] S. Giovanazzi, Phys. Rev. Lett. 94, 061302 (2005).

[11] T. G. Philbin, C. Kuklewicz, S. Robertson, S. Hill, F. König, and U. Leonhardt, Science 319, 1367 (2008).

[12] B. Horstmann, B. Reznik, S. Fagnocchi, and J. I. Cirac, Phys. Rev. Lett. 104, 250403 (2010).

[13] K. Kanti Nandi, Y.-Z. Zhang, and R.-G. Cai, arXiv:grqc/0409085.

[14] C. Sabín, Universe 4, 137 (2018).

[15] M. Kadic, G. Dupont, S. Enoch, and S. Guenneau, Phys. Rev. A 90, 043812 (2014).

[16] D. E. Fernandes, N. Engheta, and M. G. Silveirinha, Phys. Rev. B 90, 041406(R) (2014).

[17] C. Sabín, Phys. Rev. D 94, 081501(R) (2016).

[18] J. B. Pendry, D. Schurig, and D. R. Smith, Science 312, 1780 (2006).

[19] U. Leonhardt, Science 312, 1777 (2006).

[20] U. Leonhardt and T. G. Philbin, New J. Phys. 8, 247 (2006).

[21] J. Li and J. B. Pendry, Phys. Rev. Lett. 101, 203901 (2008).

[22] Y. Lai, J. Ng, H. Y. Chen, D. Z. Han, J. J. Xiao, Z.-Q. Zhang, and C. T. Chan, Phys. Rev. Lett. 102, 253902 (2009).

[23] H. Chen, C. T. Chan, and P. Sheng, Nat. Mater. 9, 387 (2010). 
[24] T. Ergin, N. Stenger, P. Brenner, J. B. Pendry, and M. Wegener, Science 328, 337 (2010).

[25] M. W. McCall, A. Favaro, P. Kinsler, and A. Boardman, J. Opt. 13, 024003 (2010).

[26] T. Ergin, J. Fischer, and M. Wegener, Phys. Rev. Lett. 107, 173901 (2011).

[27] Y. Luo, D. Y. Lei, S. A. Maier, and J. B. Pendry, Phys. Rev. Lett. 108, 023901 (2012).

[28] H. Chen, B. Zheng, L. Shen, H. Wang, X. Zhang, N. I. Zheludev, and B. Zhang, Nat. Commun. 4, 2652 (2013).

[29] R. C. Mitchell-Thomas, T. M. McManus, O. Quevedo-Teruel, S. A. R. Horsley, and Y. Hao, Phys. Rev. Lett. 111, 213901 (2013).

[30] T. Han, X. Bai, D. Gao, J. T. L. Thong, B. Li, and C.-W. Qiu, Phys. Rev. Lett. 112, 054302 (2014).

[31] S. A. R. Horsley, M. Artoni, and G. C. La Rocca, Nat. Photonics 9, 436 (2015).

[32] E. E. Narimanov and A. V. Kildishev, Appl. Phys. Lett. 95, 041106 (2009).

[33] Q. Cheng, T. J. Cui, W. X. Jiang, and B. G. Cai, New J. Phys. 12, 063006 (2010).

[34] H. Chen, R.-X. Miao, and M. Li, Opt. Express 18, 15183 (2010).

[35] C. Sheng, H. Liu, Y. Wang, S. N. Zhu, and D. A. Genov, Nat. Photonics 7, 902 (2013).

[36] A. Greenleaf, Y. Kurylev, M. Lassas, and G. Uhlmann, Phys. Rev. Lett. 99, 183901 (2007).

[37] I. I. Smolyaninov and E. E. Narimanov, Phys. Rev. Lett. 105, 067402 (2010).
[38] I. I. Smolyaninov, E. Hwang, and E. Narimanov, Phys. Rev. B 85, 235122 (2012).

[39] V. Ginis, P. Tassin, B. Craps, and I. Veretennicoff, Opt. Express 18, 5350 (2010).

[40] C. Sheng, R. Bekenstein, H. Liu, S. Zhu, and M. Segev, Nat. Commun. 7, 10747 (2016).

[41] F. Zhong, J. Li, H. Liu, and S. Zhu, Phys. Rev. Lett. 120, 243901 (2018).

[42] X. Wang, H. Liu, C. Sheng, and S. Zhu, J. Opt. 20, 024015 (2018).

[43] C. Sheng, H. Liu, H. Chen, and S. Zhu, Nat. Commun. 9, 4271 (2018).

[44] V. H. Schultheiss, S. Batz, A. Szameit, F. Dreisow, S. Nolte, A. Tünnermann, S. Longhi, and U. Peschel, Phys. Rev. Lett. 105, 143901 (2010).

[45] V. H. Schultheiss, S. Batz, and U. Peschel, Nat. Photonics 10, 106 (2015).

[46] R. Bekenstein, Y. Kabessa, Y. Sharabi, O. Tal, N. Engheta, G. Eisenstein, A. J. Agranat, and M. Segev, Nat. Photonics 11, 664 (2017).

[47] J. Zhu, Y. Liu, Z. Liang, T. Chen, and J. Li, Phys. Rev. Lett. 121, 234301 (2018).

[48] T. Roger, C. Maitland, K. Wilson, N. Westerberg, D. Vocke, E. M. Wright, and D. Faccio, Nat. Commun. 7, 13492 (2016).

[49] R. Beravat, G. K. L. Wong, M. H. Frosz, X. M. Xi, and P. S. J. Russell, Sci. Adv. 2, e1601421 (2016).

[50] E. Lustig, M.-I. Cohen, R. Bekenstein, G. Harari, M. A. Bandres, and M. Segev, Phys. Rev. A 96, 041804(R) (2017).

[51] M. S. Morris and K. S. Thorne, Am. J. Phys. 56, 395 (1988).

[52] T. Müller, Phys. Rev. D 77, 044043 (2008). 